POS PROCEEDINGS

\title{
How to best use SALT: maximising your chances
}

\section{Petri Väisänen*, Steve Crawford, Éric Depagne, Christian Hettlage, Alexei Kniazev, Paul Kotze, Brent Miszalski, Encarni Romero-Colmenero}

E-mail: petri@saao.ac.za

SALT Astronomy Operations Team, South African Astronomical Observatory and Southern African Large Telescope, South Africa

\begin{abstract}
There are a number of unique observational features due to the SALT design that the users must know to make the most of its capabilities. It also makes sense for both the PIs and for SALT as a whole to understand where SALT's strengths lie to maximise scientific productivity of programs. And it is important to be able to maximise the chances of getting those observations done once they are accepted and in the queue. Diffuse low surface brightness targets and high time/spectral resolution objects make use of the Sutherland dark background in the modest seeing conditions, while spectroscopic surveys in general fit SALT's operating mode well. Some other important aspects maximising one's chances include making use of pools of optional targets, making use of bright time, which is under-subscribed, as well as P4 filler time, and carefully considering both seasonal and nightly visibilities and track times.
\end{abstract}

SALT Science Conference 2015 -SSC2015-

1-5 June, 2015

Stellenbosch Institute of Advanced Study, South Africa

\footnotetext{
* Speaker.
} 


\section{SALT Track Times}

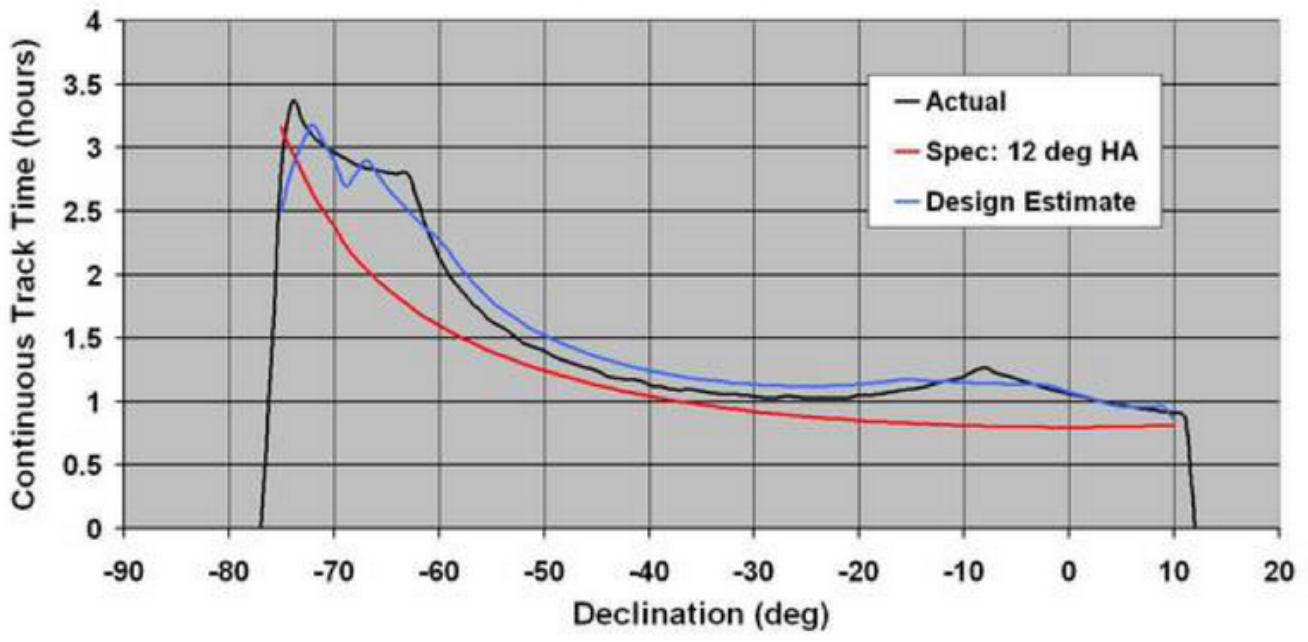

Figure 1: While objects can be visible for many hours continuously, the time of a single track (shown by the black curve) may be shorter. At Equatorial Declinations one is able to re-point the telescope in azimuth to continue for another track during the same visibility.

\section{Introduction}

The Southern African Large Telescope is a 10-m class observing facility [2], with a variety of instrument modes to use. It is fully queue scheduled. It has peculiarities with object visibility due to its fixed-altitude design.

The Sutherland observing site is very dark. Recent measurements show an average V-band sky-brightness, scaled to Zenith at Solar minimum, of around $21.9-22.0 \mathrm{mag} / \mathrm{sq} . \operatorname{arcsec}$, on a par with the very darkest sites on the planet [1]. Sutherland, however, is not an exceptionally good seeing site. Median V-band zenith seeing over the past few years is approximately $1.4 \operatorname{arcsec}$ (see also [3]), which translates to a FWHM of about 1.7 arcsec at the SALT focal plane.

Knowing how SALT works, and appreciating the instrumental, operational, and site characteristics will allow the user to make the most of SALT observations.

\section{Visibilities, blocks, and too-tight-tracks}

The altitude restrictions on SALT (47 to $59 \mathrm{deg}$ ) place observing constraints in terms of instantaneous sky access in Hour Angle and Declination. Targets are restricted to the range of Dec $=$ +11 to $-76 \mathrm{deg}$, and the continuous visibility of a target is Declination dependent and ranges from approximately $1 \mathrm{~h}$ to $4 \mathrm{~h}$. Visibility should not be confused with track time, however, which may be different depending on Dec (see Fig. 1). It is the track time that defines the maximum continuous time a target is available without re-positioning of the telescope in azimuth. For example, near the Equator visibility is long while an individual track time is about an hour (and less for an effective exposure time due to setup overheads).

All observations are defined as Blocks whose lengths must be limited to available track times. A given block, the minimum schedulable unit at SALT, can be visited multiple times, of course. 
One block will have one target and one acquisition, and while it can include more than one instrument configuration, simply designed blocks are often the most effective. When designing the blocks, it is crucial to play with the latest versions of proposal tools, in particular the PI Proposal Tool (PIPT). The tool has, for example, capabilities to visualise the time breakdown ("timeline") of the block with the various overheads for acquisition, readouts, configuration changes and calibrations.

It is important for the user to realise the implications of these characteristics. Consider what happens if the PI defines the length of her block to be very close to the available track time for those target coordinates: not only does it drastically reduce the probability of having the target observed, but also hurts the chances of others. As illustrated in Fig. 2., blocks drift through the SALT visibility annulus as the night progresses. At any given time the observer selects from the available targets the one with the highest "block score", based on an algorithm taking into account the Scientific Priority ( $\mathrm{P} 0$ being the highest, to $\mathrm{P} 4$ ) and also regarding the future opportunities of getting it and the whole program done. This is a complex calculation, and the observer attempts to optimise the night with a maximum number of high priority blocks done. Not surprisingly, those blocks with more flexibility will have higher chances of getting done. The green P2 block indicated in Fig. 2 can only be done if the telescope is free and ready to point within a 1-2 minute window. In other words, if the observer would like to get it done, the schedule of the whole night would need to built around this one block. With constantly changing weather conditions this becomes highly unlikely. The short blue blocks in this case, on the other hand, can be accessed very flexibly over several hours, making the likelihood of getting them completed over several nights very high. Using the SALT Visibility Tool is important in order to understand the visibilities and track-times for given coordinates.

\section{On-sky distribution and semester simulations}

SALT Operations can actually predict a semester fairly well by running Monte Carlo simulations mimicking the way observations are done using the real science queue with random (but statistically known) weather and other downtime patterns. It turns out that the most significant factor of not finishing high priority blocks during semester is their non-uniform distribution on the sky. The sky distribution is illustrated in Fig. 3.

In fact, our simulations show that we cannot finish all allocated blocks even if a semester had zero downtime. The target visibilities overlap too much, and sometimes also have too tight atmospheric constraints for their own good. Target distributions are difficult to rectify, since it would require coordination between the Time Allocation Committees which act independently across the partners. However, to increase the chances of an individual PI, if the science pursued allows it, one should choose objects in less oversubscribed regions in the RA/Dec space. SALT Ops will publish both phase- 1 and phase- 2 target distributions for the information of the PIs. In addition, we have added capability on the Web Manager of each accepted program to track the chances of a given block to get done. This "probability" number takes into account Moon phase and Seeing constraints, how tight the requested track is within its visibility window, and competition from all other blocks in that same window taking into account their Priorities. In case of multiple visits the number refers to the binomial probability of finishing all the requested visits taking into account the 


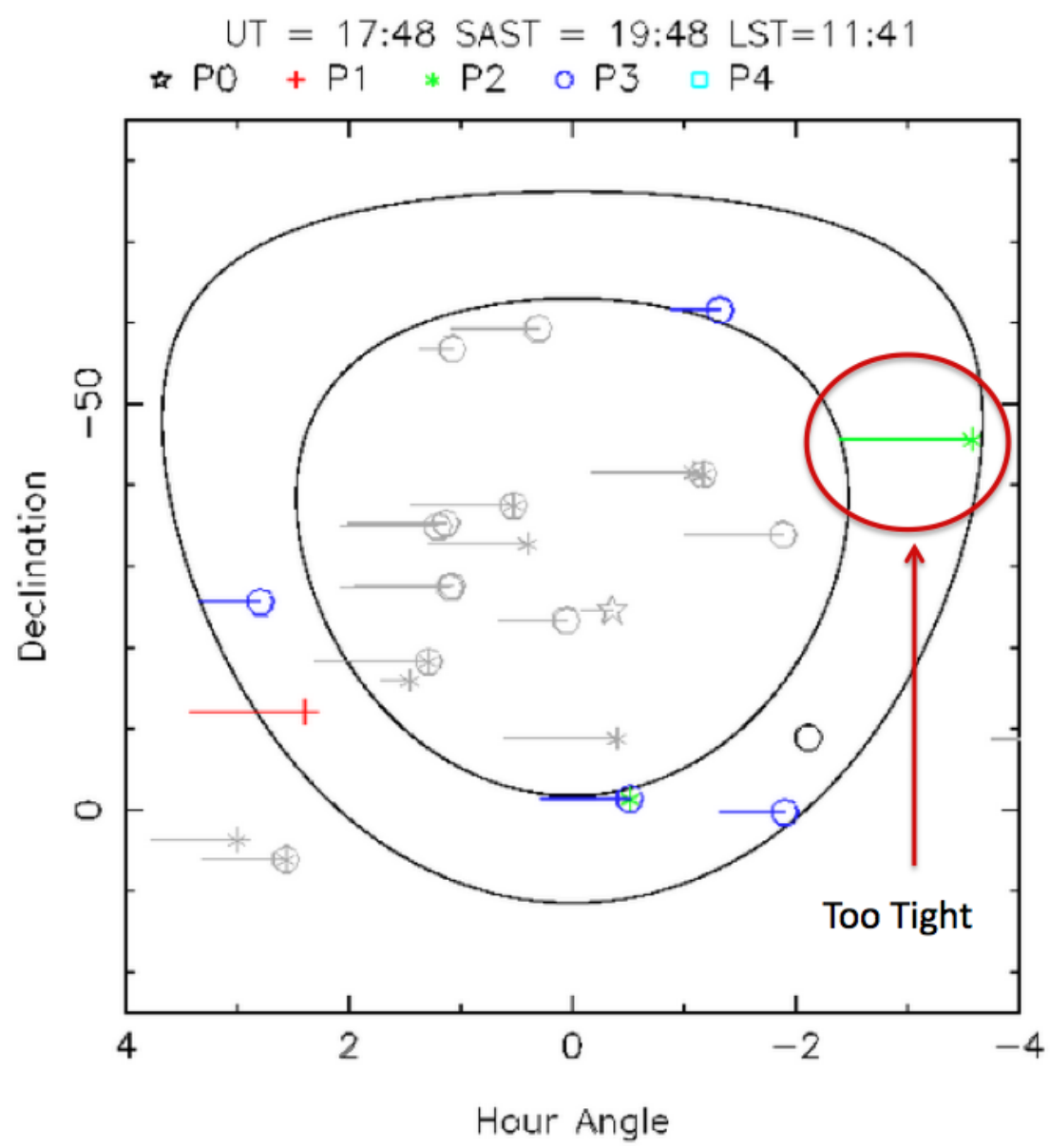

Figure 2: A SALT "toilet-seat diagram", showing active blocks at a given time drifting through the on-sky visibility annulus. The length of the lines denotes the time it takes to execute that block. The Priority 2 block indicated is too tight. While it formally fits its track time, it is extremely inflexible, there is only a 1-2 min window during the night to point to it. Hence it has a low probability of ever getting done.

available tracks over the semester. Changing e.g. observing conditions will result in live adjusting of the probability so that the PI may attempt to maximise their strategy.

\section{Optional targets, bright moon, and P4 targets}

There are other ways of increasing the chances of getting observations done in the SALT queue. One such effective way is to use optional targets, or Pools in the PIPT terminology. PIPT allows you to submit more blocks than your time allocation. This is especially useful if you have a target list with a wide RA-range. For example, if you have been allocated time to observe 10 galaxies, but actually have a larger sample and do not care which ones get observed, you should consider submitting a several times larger sample of them. This is a classic win-win situation: you greatly increase your chances of getting the required number of 10 targets done since there is often one available in the SALT visibility annulus. And simultaneously SALT Operations, in their aim to 


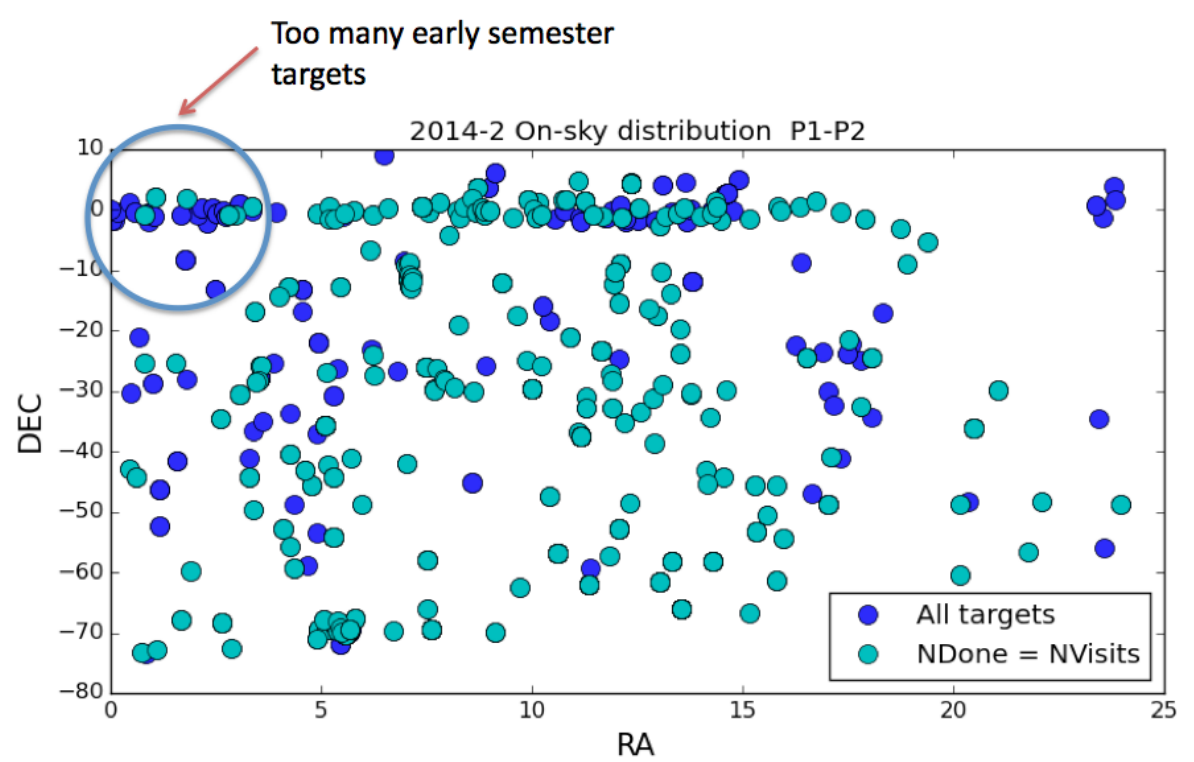

Figure 3: The on-sky distribution of P1-P2 blocks from the 2014-2 semester, the cyan ones were observed whereas the dark blue points were not done by the end of the semester. Most of them appear in regions where the TACs have just allocated too many targets for SALT to be physically able to finish. Note especially a an Equatorial group of blocks right at the beginning of observing season setting too early to have a real opportunity.

increase efficiency of observations, have a more flexible queue to operate with and can also avoid unfortunate times of no suitable targets in view.

To avoid times of an empty queue SALT allows so called P4 programs. P4 time is not charged, it does not come from any partner's time allocation. It is "free". In fact, SALT Ops also allows any accepted program to submit $\mathrm{P} 4$ filler blocks to the queue in addition to their TAC allocated higher priority blocks. These $\mathrm{P} 4$ targets in the queue are those to which we turn at moments when there is nothing else to observe. Sometimes this happens because of poor weather conditions, cloud or seeing, and only bright targets can be done. But a gap in the queue also often happens merely because of the non-uniform target distribution referred to above, or when the observer decides to wait for 15-30 minutes for a high priority target rather than starting another long one which would result in missing the high priority target. Approximately $10 \%$ or more of all observing time, some 40 to 80 hours of $\mathrm{P} 4$ time has been observed per semester in recent times.

For the reasons above, the most successful P4 targets and programs have these characteristics (though these are not requirements for them): short blocks, say, 15-30 min long which are easy to plug into gaps; bright targets, say, 10-17 mag, easily done in any conditions; a large pool of targets over a wide RA-range to have something available at any time.

Finally, for the past several years especially Bright time has been chronically undersubscribed. If one can think of useful science to be done in close to full Moon conditions, chances of getting those observations done are high merely due to less competition. Similarly, conditions when seeing is 2.5" or worse (which does happen in Sutherland), are often undersubscribed. 


\section{What is SALT especially suited for?}

It is important to try to maximise the effectiveness of SALT science by understanding its strengths, and being aware of the limitations. While the Sutherland site is very dark, seeing is only modest. In addition, until mid-2016 at least, the telescope operates in open loop alignment of the primary mirror, meaning the image quality is not competitive for doing science with faint point source targets below the sky background. Conversely, the strengths that the users should try to capitalise on, are the following:

- The large collecting power and dark skies mean that diffuse low surface brightness objects are ideal for very competitive results.

- Likewise, brighter objects where most of the light is above background regardless of the imperfect Point Spread Function, can be observed very efficiently.

- SALT is first and foremost a spectroscopic telescope. And, due to queue scheduling and the design feature of the visibility of targets, it is most efficiently used for programs doing surveys of targets over large RA-ranges.

The above does not mean that SALT absolutely cannot be used in other ways, as a totally general purpose telescope. But it does highlight the fact that overall, on average, effort should concentrate on science which can both be done efficiently and competitively for the aperture size SALT has.

Note also, that there are several modes of spectroscopy available, including multi-object and Fabry-Perot capabilities and polarimetric modes from 2016 onward, and some of these observing modes are rare on large telescopes. SALT is also capable of changing modes and instruments on-the-fly in less than a minute, making it one of the most flexible large telescopes anywhere.

\section{SALT status}

Though SALT first light happened as far back as 2005, normal science operations started in late 2011 after the main initial problems with the Spherical Aberration Corrector were fixed. There has been a steady increase in operational and technical efficiency since then, as highlighted in this Conference. Many users have heard of past problems with the spectrograph throughput - these are mostly fixed now, efficiency redward of $4000 \AA$ is close to what it should be and stray light issues affecting background have also been fixed. Technical downtime is now at a $6 \%$ level and apart from RSS polarimetric commissioning still ongoing at the time or writing, all other main modes of operation are in regular use.

There are more than 130 refereed SALT papers published, with the numbers increasing at a similar pace to other major observatories counting from the start of science operations, with decent citation counts as well. In particular, SALT papers are produced at a world record rate if normalised by operating costs: see [4] in these Proceedings for more details.

Looking into the future, active alignment of the primary mirror segments should come live in mid-2016, and SALT is getting a much improved guide probe to use with RSS by early 2017. It 
will make guidance and focusing significantly more efficient and especially help with long MOS exposures. During 2018 SALT is expecting a NIR arm for the RSS (see [5] in these Proceedings) giving $\mathrm{J}$ and $\mathrm{H}-$ band capability with an IFU unit.

\section{Summary}

The 10-m class SALT is a fixed altitude queue scheduled telescope situated at the dark Sutherland site in South Africa. Due to its design there are a number of differences how observations are conducted compared to "normal" fully steerable telescopes, and which kind of programs are most efficient. It is important for the users to understand the concepts of visibilities, track times, and blocks for useful and successful observations. For best science results it is also crucial to realise the strengths of SALT, especially with faint diffuse objects, or brighter stars above the sky background and high time or spectral resolution observations. There are several "tricks" to remember to maximise the chances of getting the most of your observations: these include the liberal use of optional targets, being mindful of too tight observing windows, capitalising on undersubscribed observing conditions and also of filler programs.

In 2015 SALT is performing well, with all its main observing modes operational and active alignment of the primary arriving in 2016, and a healthy number of science papers produced by the SALT user community.

Please do not hesitate to contact SALT Astro Ops at salthelp_at_salt.ac.za with any questions.

Acknowledgements: The SALT Astronomy Operations team wishes to thank the SSC2015 organisers for a great conference highlighting the interesting ongoing SALT science. We also acknowledge the support from SALT Foundation and the National Research Foundation of South Africa, and the many enthusiastic users of SALT.

\section{References}

[1] Benn, R.C. \& Ellison, S.L., 1998, NewAR, 42, 503

[2] Buckley, D.A.H., Swart, G., Meiring, K., 2006, SPIE, 6267, 32

[3] Catala, L., et al. 2013, MNRAS, 436, 590

[4] Schröder, A., Väisänen, P., Crawford, S.,M. 2016, these proceedings

[5] Wolf, M., 2016, these proceedings 